\title{
ACTIVE DISTURBANCE REJECTION CONTROL FOR HEAVY CARGO AIRDROP OPERATIONS
}

\author{
Shiwei Zhao - Xiuxia Sun* - Ri Liu - Dong Wang \\ Aeronautics and Astronautics Engineering College, Air Force Engineering University, Xi'an, 710038, P.R. China
}

\begin{tabular}{l} 
ARTICLE INFO \\
\hline Article history: \\
Received: 30.11 .2015$. \\
Received in revised form: 12.3 .2016$. \\
Accepted: 16.3 .2016$. \\
\hline Keywords: \\
Airdrop operations \\
nonlinear state error feedback \\
Active disturbance rejection control \\
Flight safety \\
Robustness
\end{tabular}

\section{Introduction}

Heavyweight airdrop plays an increasingly vital role in modern warfare by virtue of superiority in many aspects, such as avoiding enemy radar detection, improving the accuracy of the cargo delivery and reducing damage risk of the payloads [1-2]. During the airdrop process, the pitch angle of the carrier aircraft rises constantly under the stress of pitch moment exerted by the gradual rearward goods and followed by a fierce bow because of a sudden extraction of the cargo, which leads to sensitive flight parameters to whatever disturbance, and even to a fatal crash. Therefore, an effective controller for the airdrop is crucial to both flight safety and task performance.

Over the last few decades, many significant achievements have been made by a number of scholars: variable structure control has advantage of insensitivity over parameter changes and disturbances, removal of needs for on-line

\begin{abstract}
:
In view of the strong nonlinear and coupling characteristics of the airdrop operations, a novel control method is proposed based on the active disturbance rejection control (ADRC) for decoupling control strategy of the pitch angle and airspeed. The unknown disturbances, including aerodynamic uncertainty and nonlinear coupling effect between the aircraft and cargo dynamics, are estimated and compensated with the extended state observer (ESO). Meanwhile, the nonlinear law state error feedback (NLSEF) is adopted to restrain the compensation residual. Simulation and flight quality evaluation shows the satisfactory capacity and strong robustness of the proposed control method in guaranteeing the airdrop task and flight safety.
\end{abstract}

identification [2-3], and simplicity in mechanical implementation. But the "upper boundary" of the controller is chosen according to the "worst case", which always results in the conservation and flutter of the controller. Feedback linearization transfers the complex nonlinear model to the simple one, and achieves the decoupling control [4]. However, this method depends on the accurate knowledge about the aircraft dynamics. It is not the case with the heavyweight airdrop project since there are always some unmolded dynamics. $H_{\infty}$ control and neural networks, in theory, could obtain a perfect performance, and don't rely on the precise model of the system [5-6]. But their control effects will deteriorate constantly with the growth of the cargo weight. Under this situation, a controller should be designed by paying attention to the physical implementation, meeting the airdrop task demand and satisfying the flight safety requirement.

Active disturbance rejection control (ADRC) is a nonlinear method proposed by professor Han [7]. 
This method employs the kernel of PID controller that adopts error-feedback to regulate the system. Various disturbances both external and interior ones are estimated and compensated for properly through the ADRC controller. Numerous successful engineering applications using ADRC control have demonstrated its efficiency [7-8]. The controller proposed in this paper does not only provide a method to achieve the height maintaining and speed holding during airdrop, but also offers good system stability in the presence of uncertainty.

\section{Aircraft-cargo dynamics}

The force analysis of the transport during airdrop process is shown in Fig. 1. The longitudinal motion equation of the aircraft can be described by relations in [9]:

$$
\begin{gathered}
\dot{V}=\left(T \cos \alpha-D-m_{\mathrm{b}} g \sin \gamma-F_{\mathrm{c} x}\right) / m_{\mathrm{b}} \\
\dot{\gamma}=\left(T \sin \alpha+L-m_{\mathrm{b}} g \cos \gamma-F_{\mathrm{c} z}\right) / m_{\mathrm{b}} V \\
\dot{q}=\left(M_{y}+M_{\mathrm{c}}\right) / I_{y} \\
\dot{\theta}=q
\end{gathered}
$$

where $m_{b}$ is the mass of the cargo, the disturbances $F_{c x}, F_{c z}$ and $M_{c}$ are the friction forces, contact force and pitch moment exerted to aircraft by the cargo. $q$ is the pitch rate, $I_{y}$ is the pitch moment of inertia. The aerodynamic pitch moment $M_{y}$, drag force $D$, lift force $L$, engine thrust $T$ can be found by:

$$
\begin{gathered}
M_{y}=\bar{q} S c_{\mathrm{A}}\left[C_{m 0}+C_{m \alpha}\left(\alpha-\alpha_{0}\right)\right. \\
\left.+C_{m q} \frac{q c_{\mathrm{A}}}{2 V}+C_{m \delta_{\mathrm{e}}} \delta_{\mathrm{e}}\right] \\
D=\bar{q} S\left[C_{D 0}+C_{D \alpha}\left(\alpha-\alpha_{0}\right)+C_{D \delta_{\mathrm{e}}} \delta_{\mathrm{e}}\right] \\
L=\bar{q} S\left[C_{L 0}+C_{L \alpha}\left(\alpha-\alpha_{0}\right)+C_{L \delta_{\mathrm{e}}} \delta_{\mathrm{e}}\right] \\
T=T_{\mathrm{m}} \delta_{\mathrm{p}}
\end{gathered}
$$

where $\bar{q}$ is the dynamic pressure, $S$ is the wing area, $\delta_{\mathrm{e}}$ is the elevator deflection, $T_{m}$ is the maximum thrust of the engine, $\delta_{\mathrm{p}} \in[0,1]$ is the throttle opening, the detailed derivation and the other symbol definition can be referred to [9].

According to theoretical mechanics, the absolute acceleration vector $\boldsymbol{a}_{\boldsymbol{c}}$ can be expanded in the windaxes frame as $\boldsymbol{a}_{\boldsymbol{c}}=\boldsymbol{i} \cdot a_{\mathrm{wx}}+\boldsymbol{k} \cdot a_{\mathrm{wz}}$ :

$$
\begin{aligned}
a_{\mathrm{w} x}= & \dot{V}+q^{2} r_{\mathrm{c}} \cos \alpha-\ddot{r}_{\mathrm{c}} \cos \alpha \\
& +\dot{q} r_{\mathrm{c}} \sin \alpha+2 q \dot{r}_{\mathrm{c}} \sin \alpha \\
a_{\mathrm{w} z}= & -V \dot{\gamma}-q^{2} r_{\mathrm{c}} \sin \alpha+\ddot{r}_{\mathrm{c}} \sin \alpha \\
& +\dot{q} r_{\mathrm{c}} \cos \alpha+2 q \dot{r}_{\mathrm{c}} \cos \alpha
\end{aligned}
$$

where $r_{c}, \dot{r}_{c}$ are distance and acceleration of the cargo to the center of gravity of the airplane, respectively [9-10].

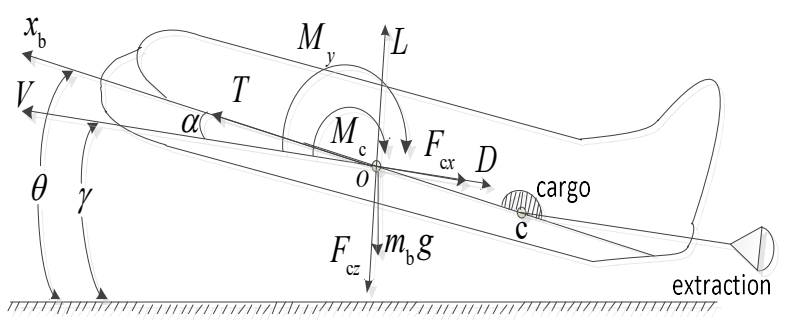

Figure 1. Graphical representation of the airdrop process and forces analysis.

The force analysis of the cargo is shown in Fig. 2. According to Newton's laws of motion, we can obtain the acceleration of cargo as:

$$
\begin{gathered}
m_{\mathrm{c}} a_{\mathrm{w} x}=F_{\mathrm{c} x}-F_{\mathrm{p}}-m_{\mathrm{c}} g \sin \gamma \\
m_{\mathrm{c}} a_{\mathrm{w} z}=-F_{\mathrm{c} z}+m_{\mathrm{c}} g \cos \gamma \\
F_{\mathrm{c} x}=\mu F_{\mathrm{c} z}
\end{gathered}
$$

where $m_{c}$ is the mass of cargo, $F_{p}=m_{c} g \lambda$ is the force of the extraction parachute with the extraction rate $\lambda, \mu$ is the friction coefficient between the cargo and the slide track.

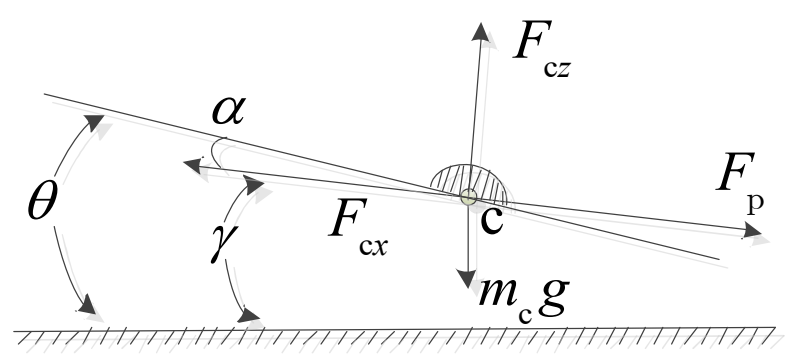

Figure 2. Forces analysis of the cargo. 
From Eqs. (9-13), we can obtain:

$$
\begin{aligned}
F_{\mathrm{c} x}= & m_{\mathrm{c}} r_{\mathrm{c}} \sin \alpha \dot{q}+m_{\mathrm{c}} \dot{V} \\
& -\left(m_{\mathrm{c}} g \cos \theta-2 m_{\mathrm{c}} q \dot{r}_{\mathrm{c}}\right) \sin \alpha \\
& +\left(m_{\mathrm{c}} g \sin \theta+m_{\mathrm{c}} q^{2} r_{\mathrm{c}}-m_{\mathrm{c}} \ddot{r}_{\mathrm{c}}\right) \cos \alpha \\
& +F_{\mathrm{p}} \\
F_{\mathrm{c} z}= & \left(m_{\mathrm{c}} g \cos \theta-2 m_{\mathrm{c}} q \dot{r}_{\mathrm{c}}\right) \cos \alpha \\
& -m_{\mathrm{c}} r_{\mathrm{c}} \cos \alpha \dot{q} \\
& +\left(m_{\mathrm{c}} g \sin \theta+m_{\mathrm{c}} q^{2} r_{\mathrm{c}}-m_{\mathrm{c}} \ddot{r}_{\mathrm{c}}\right) \sin \alpha \\
& +m_{\mathrm{c}} V \dot{\gamma} \\
\ddot{r}_{\mathrm{c}}= & \dot{V} \\
- & \cos \alpha+V \sin \alpha \dot{\gamma}+g \sin \theta \\
- & \mu(\dot{V} \cos \theta \\
+ & \mu\left(\dot{V} \sin \alpha-V \dot{\gamma} \cos \alpha+\dot{q} r_{\mathrm{c}}+2 q \dot{r}_{\mathrm{c}}\right) \\
+ & F_{\mathrm{p}} \cos \alpha / m_{\mathrm{c}}+\mu F_{\mathrm{p}} \sin \alpha / m_{\mathrm{c}}+r_{\mathrm{c}} q^{2}
\end{aligned}
$$

As a disturbance moment exerted by the cargo results from the cargo's pressure to the aircraft [9$11], M_{\mathrm{c}}$ can be described as:

$$
\begin{aligned}
M_{\mathrm{c}} & =r_{\mathrm{c}} \cdot\left(F_{\mathrm{c} z} \cos \alpha-F_{\mathrm{c} x} \sin \alpha\right) \\
& =m_{\mathrm{c}} r_{\mathrm{c}} g \cos \theta-F_{\mathrm{p}} r_{\mathrm{c}} \sin \alpha \\
& -m_{\mathrm{c}} r_{\mathrm{c}}\left(\dot{V} \sin \alpha-V \dot{\gamma} \cos \alpha+\dot{q} r_{\mathrm{c}}+2 q \dot{r}_{\mathrm{c}}\right)
\end{aligned}
$$

From Eqs. (1-8), and Eqs. (14-17), we can describe the longitudinal motion equation of the aircraft as:

$$
\dot{x}=f(x)+\Delta f(x)+(B(x)+\Delta B(x)) u
$$

where the states: $\boldsymbol{x}=[V, \alpha, q, \theta]^{\mathrm{T}}$,

the inputs: $\boldsymbol{u}=\left[\delta_{\mathrm{e}}, \delta_{\mathrm{p}}\right]^{\mathrm{T}}$,

the input-control matrix:

$\boldsymbol{B}(\boldsymbol{x})=\left[\boldsymbol{b}_{1}, \boldsymbol{b}_{2}\right]=\left[\begin{array}{llll}b_{11} & b_{21} & b_{31} & b_{41} \\ b_{12} & b_{22} & b_{32} & b_{42}\end{array}\right]^{\mathrm{T}}$,

the amount of uncertainty in the system:

$\Delta \boldsymbol{f}(\boldsymbol{x})=\left[\Delta f_{1}, \Delta f_{2}, \Delta f_{3}, \Delta f_{4}\right]^{\mathrm{T}}$.

\section{Control law design}

\subsection{Framework of the control system}

During the airdrop process, cargos are pulled out of the deck with an extraction umbrella; considering the task characteristics of ultra-low altitude heavyweight airdrop and requirements for flight safety; the flight states should be stabilized as far as possible. According to the dynamics modeling in Sec. 2, the angle of attack $\alpha$ is not affected directly by the controlled quantities $\left[\begin{array}{ll}\delta_{e} & \delta_{T}\end{array}\right]$, which is regulated with the pitch angle $\theta$ and airspeed $V$. Therefore, the controller should be designed for tracking the desired instructions of $\theta$ and $V$ to maintain the longitudinal states during the airdrop process. Meanwhile, for the outer loop, a holder for flying altitude is essential to prevent touchdown and delivery deviation. As shown in Fig. 3, the framework of the control system is integrated by the inner loops for airspeed and pitch angle stabilization and PID controller in outer loop for altitude holding.

The desired pitch angle can be obtained from the PID controller of the external loop [12-13]. In the inner loop, the airspeed and pitch angle are tracked by first-order and second-order ADRC controller, respectively, and we can obtain the desired instructions $V_{c}, \theta_{c}$ through the decoupling control.

Both the elevator deflection $\delta_{\mathrm{e}}$ and the throttle opening $\delta_{\mathrm{p}}$ have an effect on the airspeed and pitch angle at the same time. Therefore, a proper rudder distribution method is necessary for meeting both requirements, as we know:

$\left[\begin{array}{l}u_{V c} \\ u_{\theta c}\end{array}\right]=\left[\begin{array}{ll}b_{11} & b_{12} \\ b_{21} & b_{22}\end{array}\right]\left[\begin{array}{l}\delta_{e} \\ \delta_{p}\end{array}\right]$

Then,

$$
u=\left[\begin{array}{l}
\delta_{e} \\
\delta_{p}
\end{array}\right]=\left[\begin{array}{ll}
b_{11} & b_{12} \\
b_{21} & b_{22}
\end{array}\right]^{-1}\left[\begin{array}{l}
u_{V c} \\
u_{\theta c}
\end{array}\right]
$$

Through the states of feedback and disturbance compensation we can obtain the desired dynamic response. 


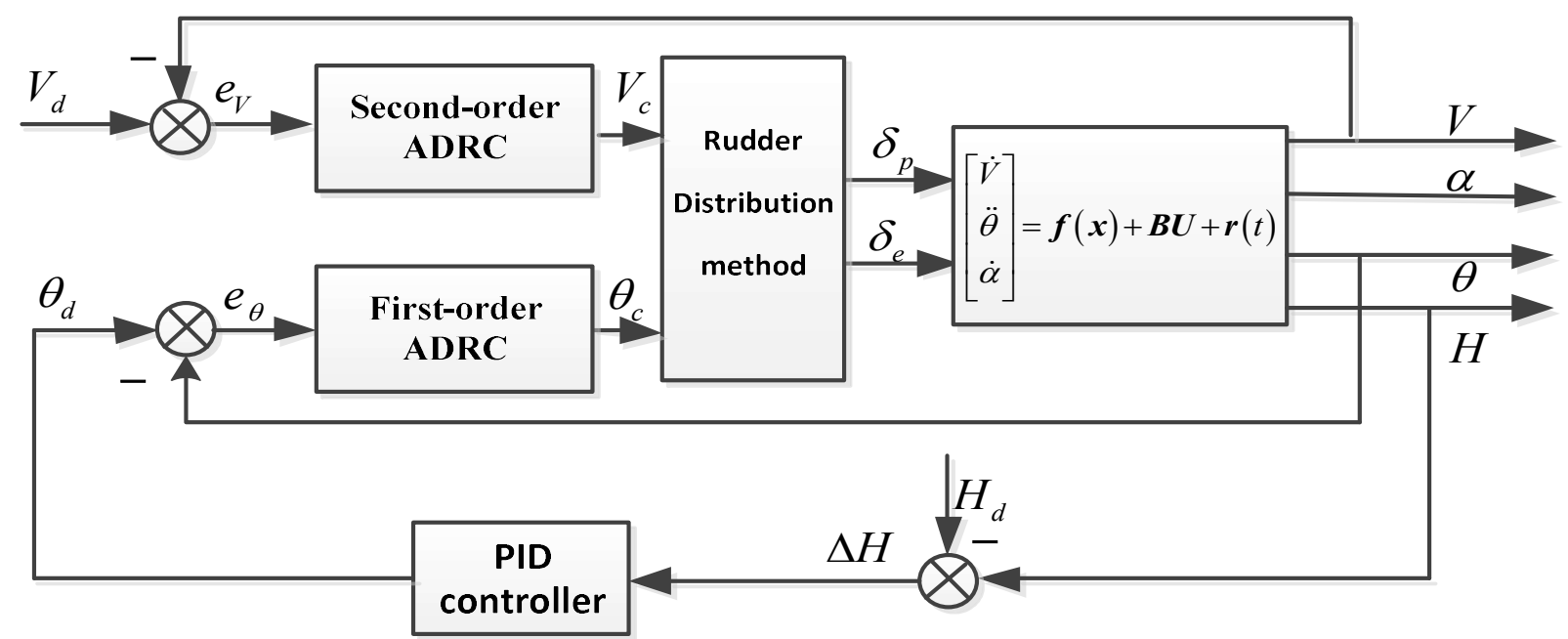

Figure 3. Framework of the control system.

\subsection{ADRC theory}

For the airspeed $V$ and the pitch angle $\theta$, firstorder and second-order ADRC controller has been designed, respectively. Since there is a similar principle for two channels, we take here the subloop of the pitch angle as an example to explain the design of the ADRC controller.

Considering the following system with uncertainty:

$\ddot{\theta}=f(\theta, \dot{\theta})+r(t)+b u$

where $r(t)$ is uncertainty, $b$ is a constant.

As illustrated in Fig. 4, the controller of the pitch angle consists of tracking differentiator (TD), nonlinear law state error feedback (NLSEF) and extended state observer (ESO). Let $\theta_{d}$ denote the trim pitch angle.

1) Tracking Differentiator (TD)

According to $\theta_{d}$, the TD produces the input of the controller:

$$
\left\{\begin{array}{l}
\dot{\theta}_{d 1}=\theta_{d 2} \\
\dot{\theta}_{d 2}=-R \cdot \operatorname{sign}\left(\theta_{d 1}-\theta_{d}+\frac{\theta_{d 2}\left|\theta_{d 2}\right|}{2 R}\right)
\end{array}\right.
$$

where $R$ is the tracking parameter, which determines the rate of the TD for tracking the input, the signal function $\operatorname{sign}(\cdot)$ is used to reduce the difference between $\theta_{d 2}$ and $\dot{\theta}_{d}$. Thus $\theta_{d 1} \rightarrow \theta_{d}$ with the transient process to prevent an excess input, and $\theta_{d 2} \rightarrow \dot{\theta}_{d}$ to provide the differential signal shielding the noise interference $[6,12]$.

2) Extended State Observer (ESO)

There are states $x_{1}=\theta, x_{2}=\dot{\theta}$, and the external disturbance $x_{3}$, which can be evaluated with the extended state observer (ESO):

$$
\left\{\begin{array}{l}
e=z_{1}-\theta \\
\dot{z}_{1}=z_{2}-b_{\theta 1} f a l\left(e, a_{\theta 1}, z_{\theta 1}\right) \\
\dot{z}_{2}=z_{3}-b_{\theta 2} f a l\left(e, a_{\theta 2}, z_{\theta 2}\right)+u \\
\dot{z}_{3}=-b_{\theta 3} f a l\left(e, a_{\theta 3}, z_{\theta 3}\right)
\end{array}\right.
$$

where $b_{\theta i}, i=1,2,3$ are feedback coefficients, $z_{1}$, $z_{2}$ are the estimations of the pitch angle and angle rate, $z_{3}$ is the estimation of "total disturbance" (the extended state) which contains the external disturbance $r_{\theta}$ and the nonlinear dynamics $f_{\theta}(\boldsymbol{x})$.

$f a l(u, a, z)=\left\{\begin{array}{ll}|u|^{a} \operatorname{sign}(u) & |u|>z \\ \frac{u}{z^{1-a}} & |u| \leq z\end{array}\right.$, with the

nonlinear exponential $a$ and saturation zone $z$ is the time optimal control function [14] to approximate the states instantly but without high frequency flutter: $z_{1} \rightarrow \theta, z_{2} \rightarrow \dot{\theta}, z_{2} \rightarrow f_{\theta}+r_{\theta}$. 
As the key of the ADRC controller, the stability of the ESO is closely related to the effectiveness of the method. The error function of second-order ESO can be described as:

$$
\left\{\begin{array}{l}
e_{1}=e_{2}-\beta_{1} f_{1}\left(e_{1}\right) \\
e_{2}=w(t)-\beta_{2} f_{2}\left(e_{1}\right)
\end{array}\right.
$$

where $w(t)$ is the change rate of external state $x_{3}$. Define the function:

$$
h\left(e_{1}, e_{2}\right)=e_{2}-\beta_{1} f_{1}\left(e_{1}\right)+k g_{1}\left(e_{1}\right) \operatorname{sign}\left(e_{1}\right)
$$

where $g_{1}\left(e_{1}\right)$ is a continuous positive definite function with $g_{1}(0)=0, k>1$ is a constant.

Define the area:

$$
G=\left\{\left(e_{1}, e_{2}\right): \mid h\left(e_{1}, e_{2}\right) \leq g_{1}\left(e_{1}\right)\right\}
$$

and assume that $\left(e_{1}(t), e_{2}(t)\right) \in G$, then when $\forall t>T$, we can obtain that:

$$
\begin{aligned}
& \dot{e}_{1}+k g_{1}\left(e_{1}\right) \operatorname{sign}\left(e_{1}\right) \leq g_{1}\left(e_{1}\right) \\
& -k g_{1}\left(e_{1}\right) \operatorname{sign}\left(e_{1}\right)-g_{1}\left(e_{1}\right) \leq \dot{e}_{1} \\
& \dot{e}_{1} \leq-k g_{1}\left(e_{1}\right) \operatorname{sign}\left(e_{1}\right)+g_{1}\left(e_{1}\right)
\end{aligned}
$$

since $g_{1}\left(e_{1}\right)>0 k>1, \forall e_{1} \neq 0$; then: if $e_{1}>0, e_{1} \dot{e}_{1} \leq(1-\mathrm{k}) g_{1}\left(e_{1}\right) e_{1}<0 \quad \forall e_{1} \neq 0$;

$$
e_{1}<0, e_{1} \dot{e}_{1} \leq(k-1) g_{1}\left(e_{1}\right) e_{1}<0 \quad \forall e_{1} \neq 0 .
$$

Define $V_{1}=\frac{1}{2} e_{1}^{2}$, then $\dot{V}_{1}=e_{1} \dot{e}_{1}<0 \quad \forall e_{1} \neq 0, t>T$; so, when $t \rightarrow \infty, \quad e_{1}(t) \rightarrow 0$; if $f_{1}(0)=0$, $e_{2}(t) \rightarrow 0(t \rightarrow \infty)$, which means that the area $G=\left\{\left(e_{1}, e_{2}\right): \mid h\left(e_{1}, e_{2}\right) \leq g_{1}\left(e_{1}\right)\right\}$ is the area within which the system later converges to its original point.

And for states out of the area $G$, the function is defined: $V_{h g}=\frac{1}{2}\left(h^{2}\left(e_{1}, e_{2}\right)-g_{1}^{2}\left(e_{1}\right)\right)$ :

when $V_{h g} \geq 0 \forall\left(e_{1}, e_{2}\right) \neq 0, \dot{V}_{h g}<0$ then states that do not converge to the original point will arrive at the boundary $V_{h g}=0$, and then penetrate into the $G$. On this basis:

Assume the change rate of the extended state $w(t)=0, f_{1}\left(e_{1}\right) \propto e_{1}$ and define $f^{\prime}=\frac{d f\left(e_{1}\right)}{d e_{1}}$. If in the system (22) $\beta_{1}^{2}>\frac{4 \beta_{2}}{f_{1}^{\prime}}\left|\left(\frac{f_{2}}{f_{1}^{\prime}}\right)\right|$, the state will be bounded within the area $G$ with the condition:

$g_{1}\left(e_{1}\right)=\frac{1}{k} \frac{\beta_{2}\left|f_{2}\right|}{\beta_{1} f_{1}^{\prime}}, 0<k<k^{*},\left(k^{*}+1\right)^{2}=4 c_{1} k^{*}$

$c_{1}=\frac{\beta_{1}^{2} f_{1}^{\prime}}{4 \beta_{2}\left|\left(f_{2} / f_{1}^{\prime}\right)^{\prime}\right|}>1$.

If the function $f_{1}\left(e_{1}\right)=\left|e_{1}\right|^{a_{1}} \operatorname{sign}\left(e_{1}\right)$, $f_{2}\left(e_{1}\right)=\left|e_{1}\right|^{a_{2}} \operatorname{sign}\left(\mathrm{e}_{1}\right)$, then for the parameters of the ESO:

$$
\begin{gathered}
\beta_{1}^{2}>\frac{4\left(1+a_{2}-a_{1}\right) \beta_{2}}{a_{1}}\left|e_{1}\right|^{1+a_{2}-2 a_{1}} \\
g_{1}\left(e_{1}\right)=\frac{\beta_{2}}{k \beta_{1}}\left|e_{1}\right|^{1+a_{2}-a_{1}}
\end{gathered}
$$

Since $g_{1}(0)=0,1+a_{2}-a_{1}>0$. If $w(t) \neq 0$ but within a finite boundary $|w(t)|<W$. Assume $f_{1}\left(e_{1}\right)=e_{1}, f_{2}\left(e_{1}\right)=f a l\left(e_{1}, a, \delta\right) \quad(0<a<1)$, and consider:

$$
\begin{gathered}
g_{1}\left(e_{1}\right)=\frac{\beta_{2}}{k \beta_{1}}\left|f_{2}\right| \\
g_{2}\left(e_{1}, e_{2}\right)= \begin{cases}\left|h_{2}\left(e_{1}, e_{2}\right)\right| & V_{h g}>0 \\
g_{1}\left(e_{1}\right) & V_{h g} \leq 0\end{cases}
\end{gathered}
$$

if $\beta_{1}^{2}>4 c_{2} \beta_{2}\left|f_{2}^{\prime}\right|, c_{2}>1$, there will be an area $G_{0}$, which contains the original point:

$$
G_{0}=\left\{\left(e_{1}, e_{2}\right): \beta_{1} g_{2}\left(e_{1}, e_{2}\right) \leq \frac{c_{2}}{c_{2}-1} W\right\}
$$

and its boundary is: $g_{2}\left(e_{1}, e_{2}\right)=\frac{c_{2}}{\beta_{1}\left(c_{2}-1\right)} W$, according to the definition of $g_{2}\left(e_{1}, e_{2}\right)$, we can obtain that:

$$
\begin{cases}\left|h_{1}\left(e_{1}, e_{2}\right)\right|=\frac{c_{2}}{\beta_{1}\left(c_{2}-1\right)} W & V_{h g}>0 \\ g_{1}\left(e_{1}\right)=\frac{c_{2}}{\beta_{1}\left(c_{2}-1\right)} & V_{h g} \leq 0\end{cases}
$$


By combining the definition of $h_{1}\left(e_{1}, e_{2}\right)$ and $\mathrm{g}_{1}\left(e_{1}\right)$, we can obtain that:

$$
\left\{\begin{aligned}
e_{1}^{*}=\max \left\{\left|e_{1}\right|\right\} & =f_{2}^{-1}\left(\frac{k c_{2} W}{\beta_{2}\left(c_{2}-1\right)}\right) \\
& =\left(\frac{k c_{2} W}{\beta_{2}\left(c_{2}-1\right)}\right)^{\frac{1}{a}} \\
e_{2}^{*}=\max \left\{\left|e_{2}\right|\right\} & =\beta_{1} e_{1}^{*}-\frac{(k-1) c_{2} W}{\beta_{1}\left(c_{2}-1\right)}
\end{aligned}\right.
$$

In Eq.(28), $e_{1}^{*}$ reflects the estimation error for the states of the system; $e_{2}^{*}$ determines the estimation error to the extended state (total disturbance), when $\beta_{2}>\frac{k c_{2} W}{c_{2}-1}, e_{1}^{*}$ and $e_{2}^{*}$ will be reduced with the diminution of $a$, which means the adaptability of the ESO to the uncertainty and finally disturbance is enhanced.

What is noteworthy here is that the error analysis mentioned above is based on a precondition $|w(t)|<W$, which is in accordance with the airdrop operations.

3) Nonlinear Law State Error Feedback (NLSEF) In the traditional control method, the control quantity $u_{c}$ usually consists of the linear combination of output error, while the nonlinear feedback is more efficient than the linear in eliminating the error [15]:

$$
\left\{\begin{aligned}
e_{1}= & \theta_{d 1}-z_{1} \\
e_{2}= & \theta_{d 2}-z_{2} \\
u_{0}= & k_{\theta 1} \cdot f a l\left(e, a_{k 1}, z_{k 1}\right) \\
& +k_{\theta 2} \cdot f a l\left(e, a_{k 2}, z_{k 2}\right)
\end{aligned}\right.
$$

where $k_{\theta 1}, k_{\theta 2}$ is the nonlinear feedback coefficient. Then

$$
u_{\theta c}=\left(u_{0}-z_{3}\right) / b
$$

where $z_{3} / \mathrm{b}$ as a feedback quantity is used to compensate for the uncertain disturbance. By combining Eq. (18) and (30), we can obtain that:

$$
\begin{aligned}
\ddot{\theta} & =f_{\theta}+b \cdot u_{\theta c}+r_{\theta} \\
& =f_{\theta}+r_{\theta}+u_{0}-z_{3}=u_{0}
\end{aligned}
$$

Thus, a nonlinear system with unknown disturbance and uncertainty is transferred into a second-order linear equation; it is well-known that the model $y^{(n)}=u_{0}$ is easy to be controlled.

As the controller designed for the whole system is independent of the accurate model, ADRC can achieve the decoupling control for all states and obtain strong robustness.

\subsection{Controller algorithm}

With the analysis above, the pitch angle control instruction from PID controller can be described as:

$$
\theta_{d}=K_{p} \Delta H+K_{I} \int \Delta H d t+K_{D} \frac{d H}{d x}
$$

Algorithms of the two sub-loops are:

1) Airspeed sub-loop

The TD for airspeed can be defined as:

$$
\left\{\begin{array}{l}
\dot{x}_{1}=x_{2} \\
\dot{x}_{2}=-R \cdot f a l\left(x_{1}-v(t)+\frac{x_{2}\left|x_{2}\right|}{2 R}, a_{T 1}, z_{T 1}\right)
\end{array}\right.
$$

where $R$ is tracking rate.

To estimate the airspeed and relevant uncertainty (extended state), the ESO is defined as:

$$
\left\{\begin{array}{l}
e=z_{1}-x_{1} \\
\dot{z}_{1}=z_{2}-b_{V 1} f a l\left(e, a_{V 1}, z_{V 1}\right)+u \\
\dot{z}_{2}=-b_{V 2} f a l\left(e, a_{V 2}, z_{V 2}\right)
\end{array}\right.
$$

where $z_{1}$ is the estimation of the system state, $z_{2}$ is the one for the extended state.

According to the state $z_{1}$ and the extended state $z_{2}$, we can design control law to compensate for the uncertainty:

$$
u_{v c}=k_{V} \cdot f a l\left(e, a_{V}, z_{V}\right)-z_{2}
$$


where $k_{V}$ is nonlinear gain of the airspeed error function $f a l\left(e, a_{V}, z_{V}\right)$.

\section{2) Pitch angle sub-loop}

For the TD algorithm of the pitch angle sub-loop, it is similar to the one in TD sub-loop.

For the pitch angle sub-loop, the pitch angle $\theta$, pitch rate $q$, and relevant uncertainty (extended state) need to be estimated by the ESO:

$$
\left\{\begin{array}{l}
e=z_{1}-\theta \\
\dot{z}_{1}=z_{2}-b_{\theta 1} f a l\left(e, a_{\theta 1}, z_{\theta 1}\right) \\
\dot{z}_{2}=z_{3}-b_{\theta 2} f a l\left(e, a_{\theta 2}, z_{\theta 2}\right)+u \\
\dot{z}_{3}=-b_{\theta 3} f a l\left(e, a_{\theta 3}, z_{\theta 3}\right)
\end{array}\right.
$$

where $z_{1}, z_{2}$ are the evaluations to the pitch angle and angle rate, $z_{3}$ is the one to extended state.

Similar to the airspeed sub-loop, the control law for the pitch angle is:

$$
\begin{aligned}
u_{\theta c}= & k_{\theta 1} \cdot f a l\left(e, a_{k 1}, z_{k 1}\right) \\
& +k_{\theta 2} \cdot f a l\left(e, a_{k 2}, z_{k 2}\right)-z_{3}
\end{aligned}
$$

where $k_{\theta 1}, k_{\theta 2}$ are the gains for error of angle and angle rate respectively.

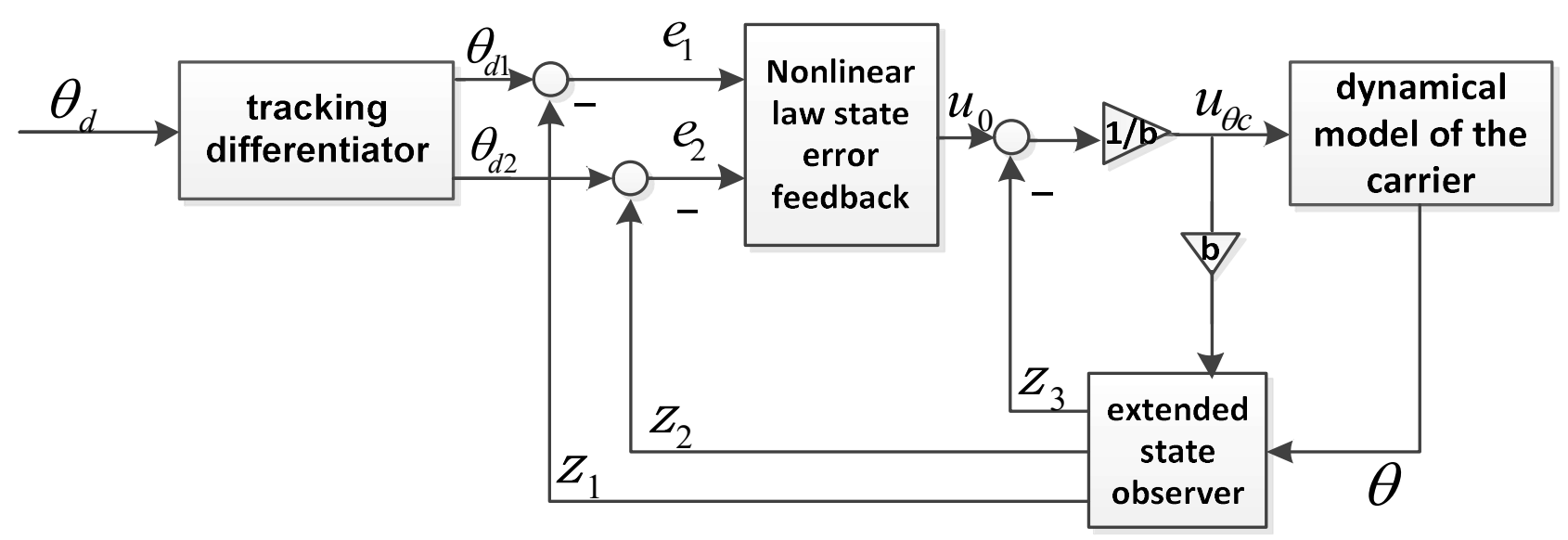

Figure 4. Second-order ADRC controller block for pitch angle.

\section{Simulation analysis}

To demonstrate the efficiency of the controller for the airdrop operations, we simulate, for example, a $24,955 \mathrm{~kg}$ transport aircraft airdrop, 4,000 kg and $8,000 \mathrm{~kg}$ payload, respectively. The initial conditions are: flight altitude $H_{0}=10 \mathrm{~m}$, airspeed $V_{0}=80 \mathrm{~m} / \mathrm{s}$, flap $\delta_{f}=25 \mathrm{deg}$, and elevator $\delta_{e}=0 \mathrm{deg}$. Firstly, the aerodynamic response of the aircraft without controller is tested to analyze the flight characteristics of the airdrop process. Then the controller performance is shown to demonstrate its capability. At last, the robustness of the controller is tested with the aerodynamic coefficients timevarying. The parameters of the controller are:
The PID parameters for external loop:

$$
K_{p}=0.05, K_{I}=0.02, K_{D}=0.005 \text {. }
$$

And within the internal loop:

a) For the TD, both airspeed and pitch angle subloop are resembled:

$R=100, a_{T 1}=0, z_{T 1}=0.0025$.

b) The other parameters are:

$a_{V 1}=0.5, z_{V 1}=0.002, a_{V 2}=0, z_{V 1}=0.0025$,

$b_{V 1}=b_{V 2}=10, a_{V}=0.5, z_{V}=0.0025, K_{V}=0.8$.

$a_{\theta 1}=0.6, z_{\theta 1}=0.0025, b_{\theta 1}=10, a_{\theta 2}=0.56$,

$z_{\theta 2}=0.0025, b_{\theta 2}=30, a_{\theta 3}=0, z_{\theta 3}=0.002$,

$b_{\theta 3}=50$. 
$a_{K 1}=0.5, z_{K 1}=0.0025, K_{\theta 1}=1.8$,

$a_{K 2}=0.5, z_{K 2}=0.0025, K_{\theta 2}=1.2$.

When the aircraft airdropping is without control, as shown in Fig. 4, the cargo's movement to the rear exerts an adverse effect on the carrier's stability, and this phenomenon is insufferable for the real airdrop task and the flight safety, which manifests the vital importance of a controller for the airdrop operation.
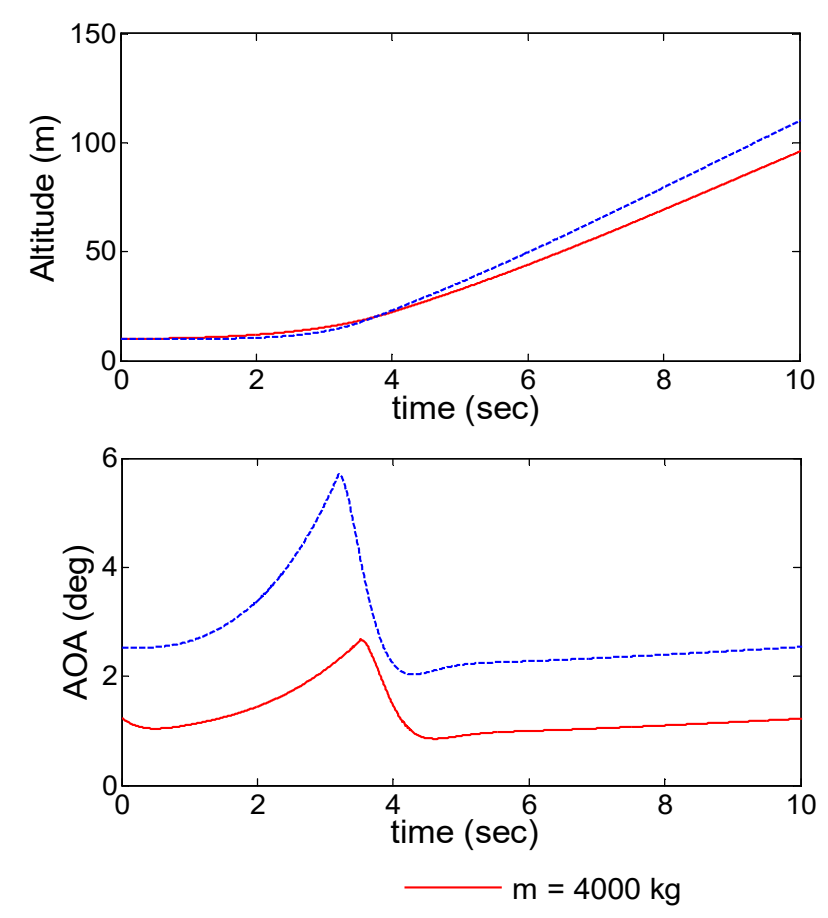

Figure 5 represents the flight states with the ADRC controller; and the result of estimation to the total disturbance (extended states) by the ESO is illustrated in Fig. 6. It can be seen that the plane tends to be steady when time is $5 \mathrm{~s}$, and all of the flight states are within the allowed range safety. Meanwhile, from the Fig. 6 we can conclude that the proposed controller is fully capable of dealing with disturbance and uncertainty, which is also the key advantage of this method compared to the other strategies.
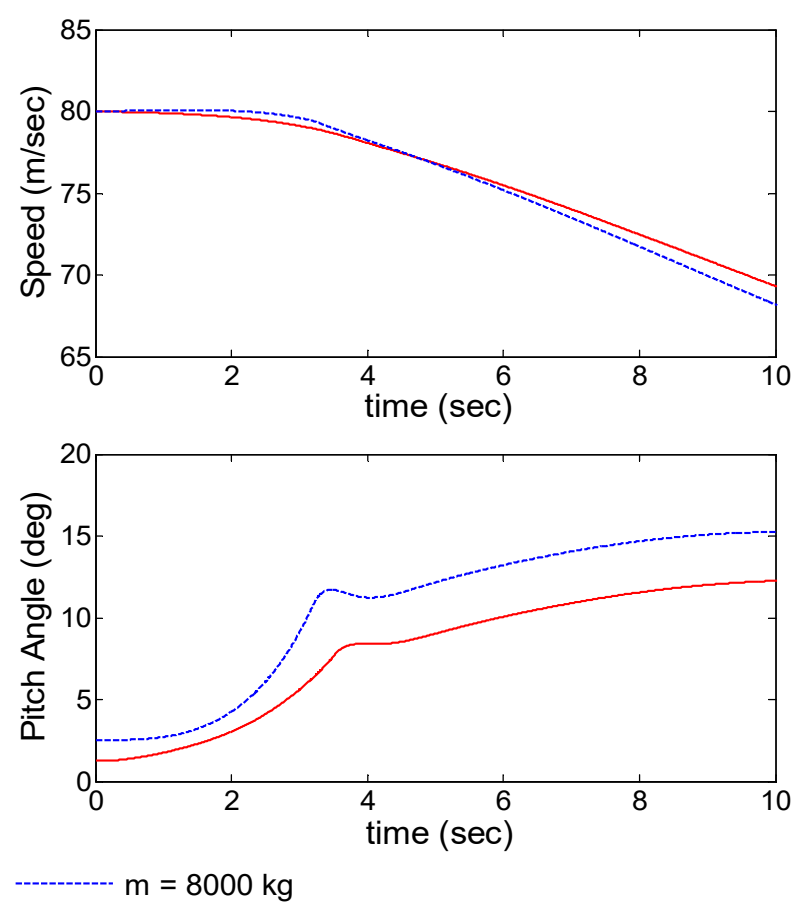

Figure 4. Longitudinal states of the aircraft without control.

During the real airdrop process, the flight control system may fail to maintain satisfactory stability or performance due to the influence of unknown disturbance, especially for the aerodynamic coefficients. In order to test the robustness of the proposed control system, as is shown in Fig. 7, we suppose that the aerodynamic coefficients have $\pm 20 \%$ uncertainty; and Fig. 8 for the system with the aerodynamic coefficients time-varying $c=c_{0} *[1+0.25 *(\pi t / 2)]$, where $c_{0}$ is the aerodynamic coefficient under the normal condition. We can see that the controller eliminates the effects of the uncertainty perfectly, which proves the ADRC controller has strong robustness against disturbance and uncertainty during the airdrop process.
To assess the effectiveness of the controller objectively, the flight quality of the aircraft should be evaluated in view of the characteristic of the airdrop task, which can be examined from two aspects:

(1) Handling quality of the aircraft.

According to the flight quality specification defined by the U.S Department of Defense [16], the handling quality of the aircraft can be evaluated with criteria of Chalk and Gibson based on the transfer functions analysis. The low-order equivalent transfer function of the pitch rate $f_{\dot{\theta}}$ can be described as: 


$$
f_{\dot{\theta}}=\frac{5.221 s^{6}+569.8 s^{5}+4896 s^{4}+13340 s^{3}+10927 s^{2}+3123 s+220.3}{s^{8}+124.9 s^{7}+25622 s^{5}+177920 s^{4}+265910 s^{3}+256690 s^{2}+64218 s+4657}
$$
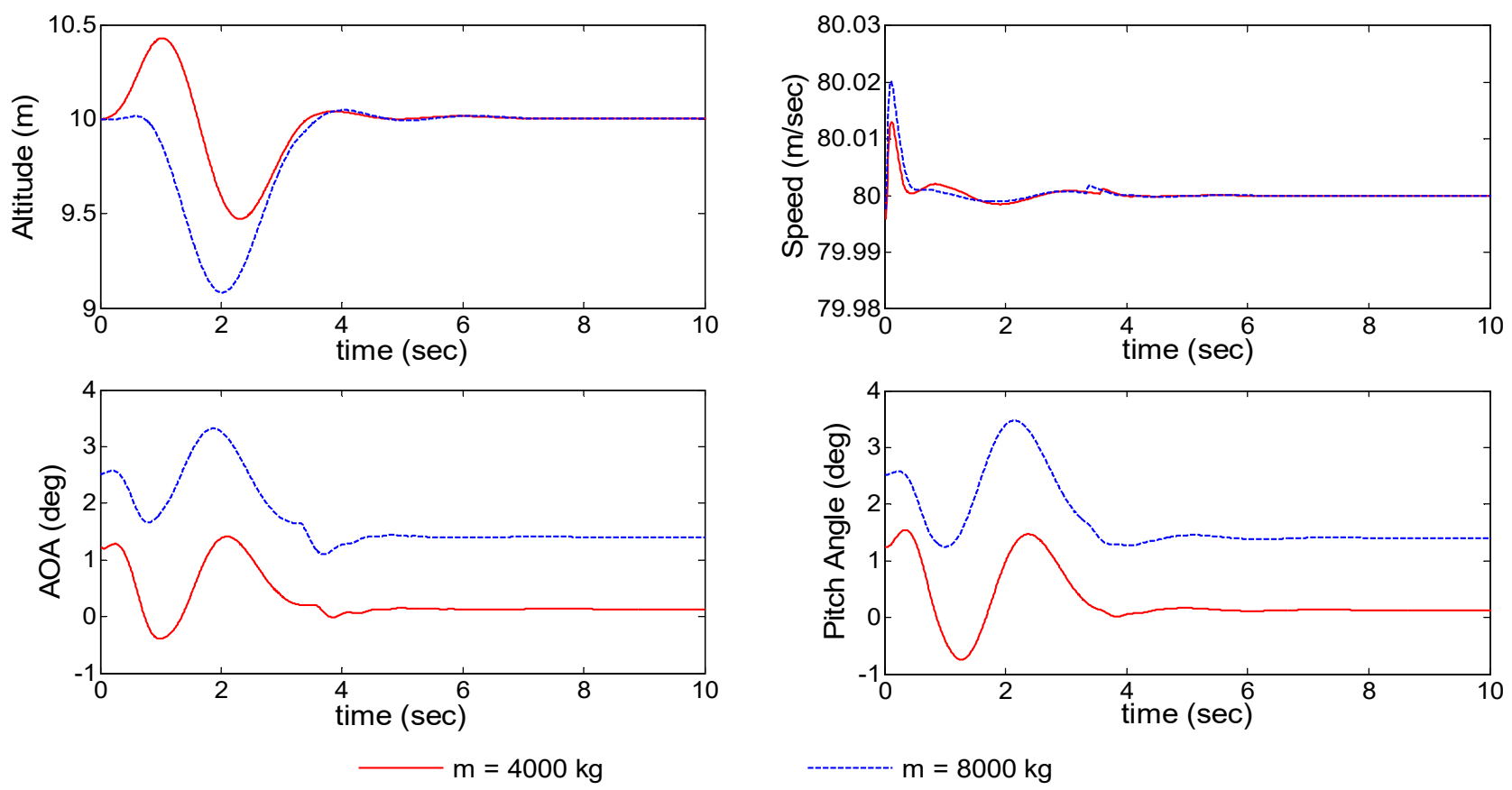

Figure 5. Longitudinal states of the aircraft under the ADRC controller.
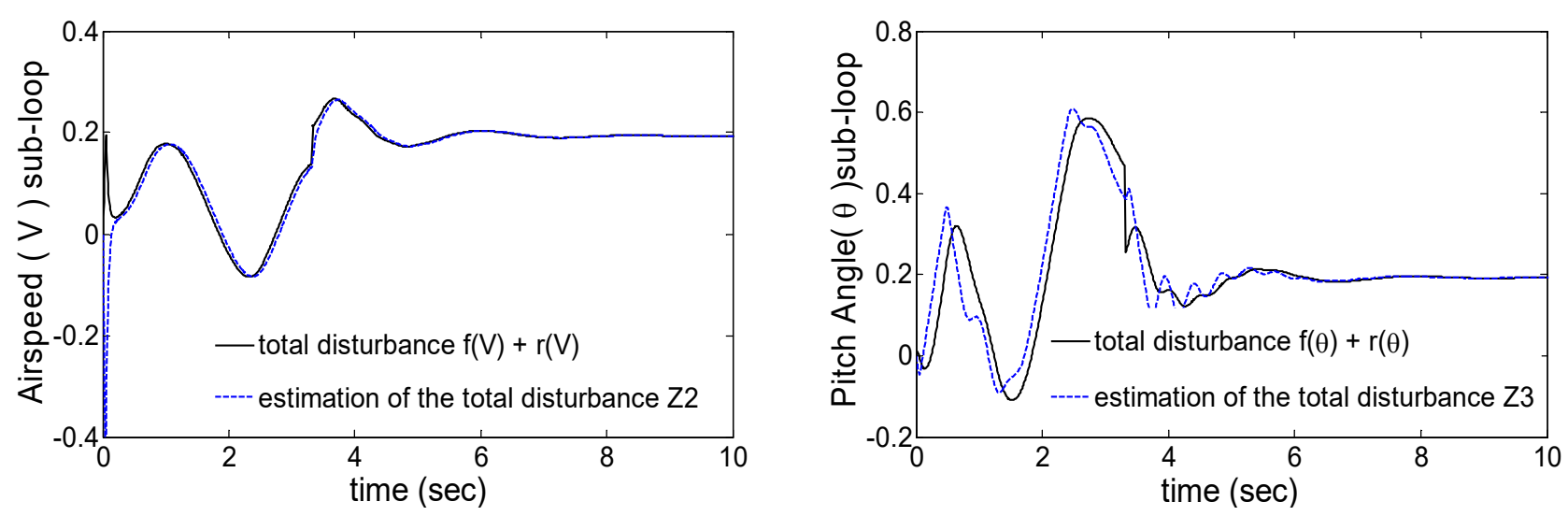

Figure 6. "total disturbance" and its estimation by ESO.

Then the result about the pitch rate response according to the Chalk Criteria is listed in Table 1. And the evaluation for pitch angle response with the Gibson Criteria is illustrated in Fig. 9 (the detail definition of the related symbol can be referred to Ref. [16]). The results show that both the pitch angle and pitch rate meet the requirement of level flight quality, which means that the handling quality of the aircraft is clearly adequate for the flight phase. 

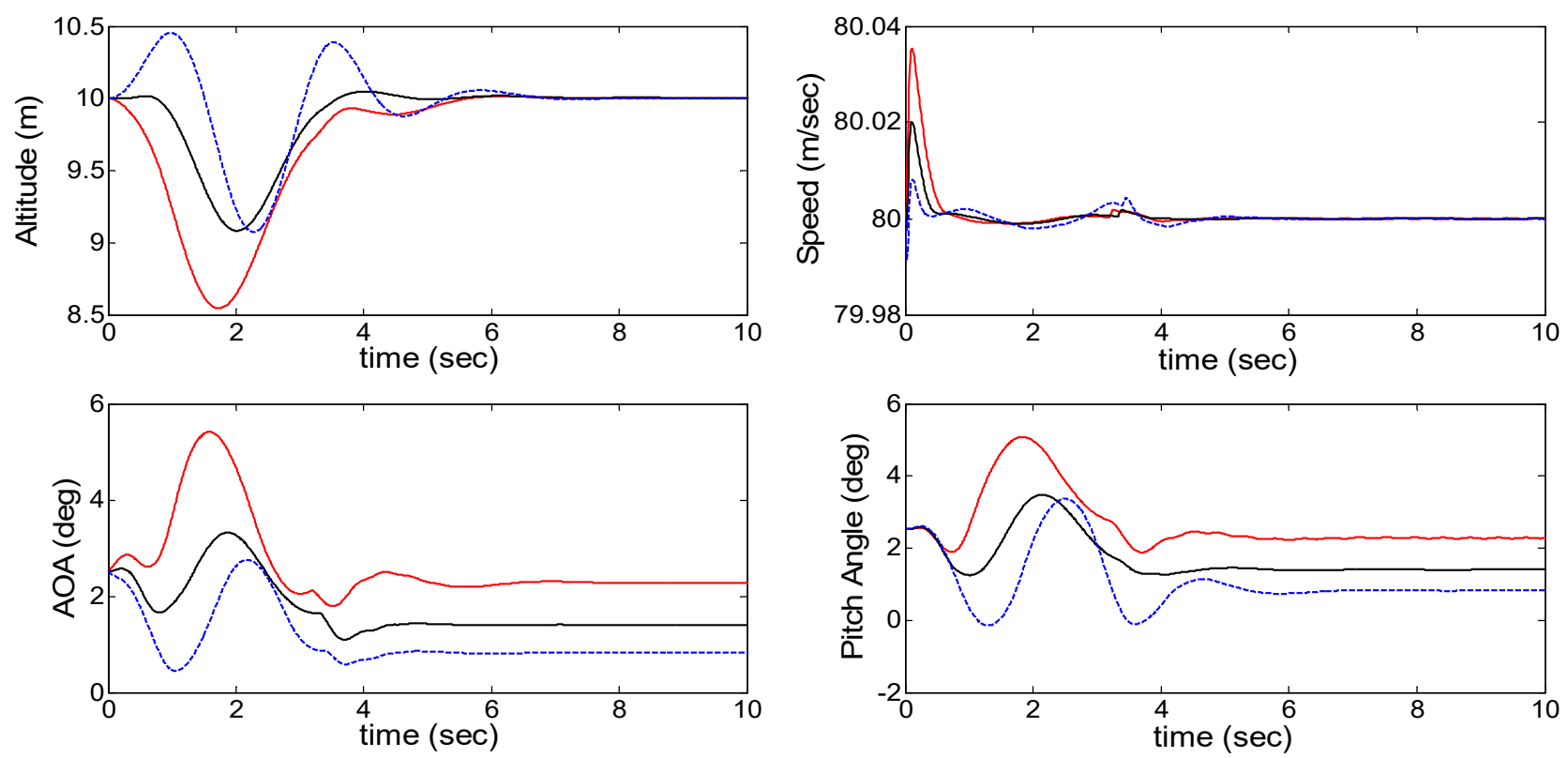

$--20 \%$ aerodynamic coefficient perturbation - normal condition ----- $+20 \%$ aerodynamic coefficient perturbation

Figure 7. Longitudinal states of the aircraft with aerodynamic coefficients perturbation.
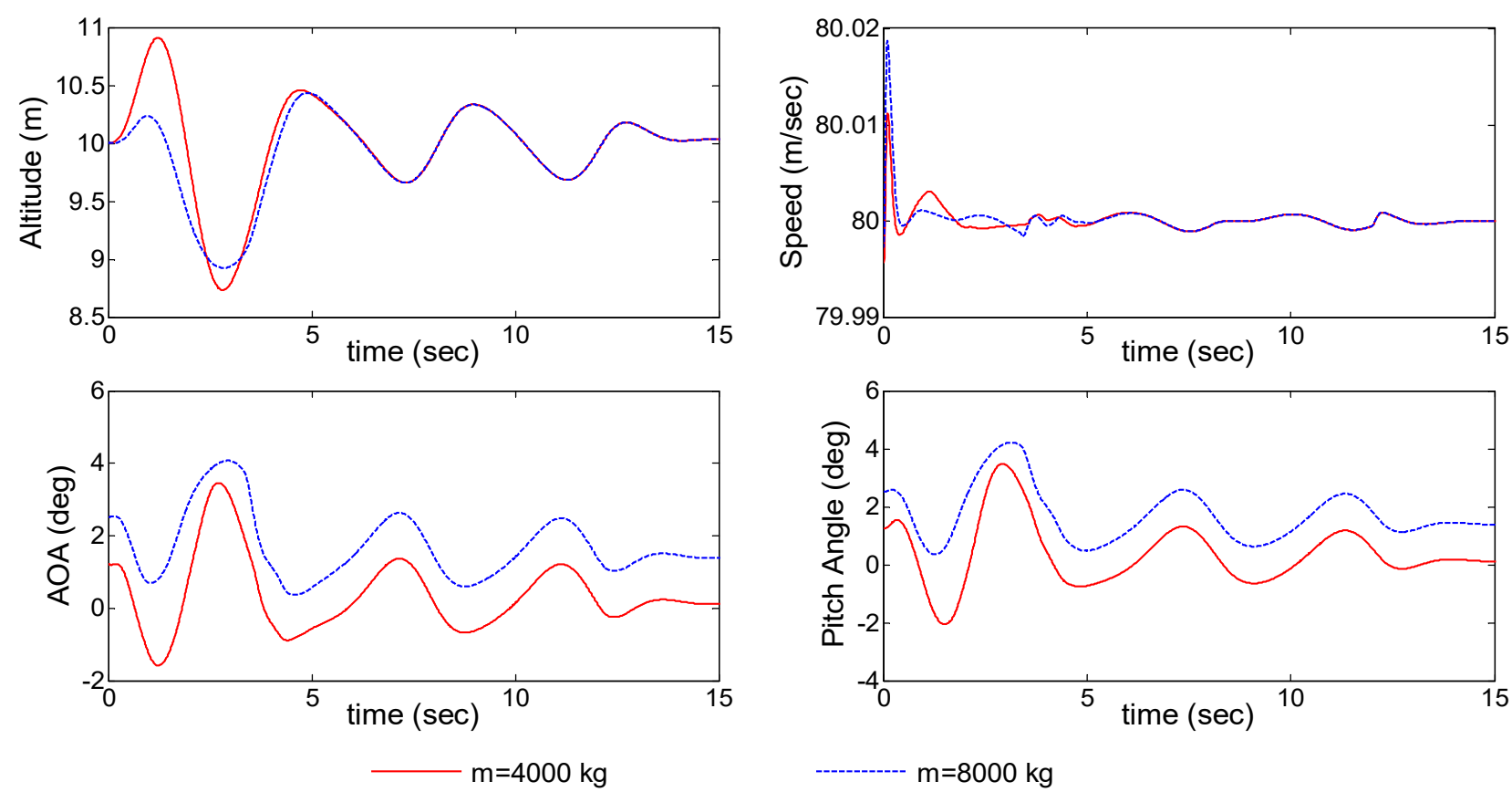

Figure 8. Longitudinal states of the aircraft with aerodynamic coefficients time-varying.

(2) Mission capability and safety evaluation

Until now, there is still no universally accepted evaluation criterion for airdropping mission and safety. On the basis of reference to the related data about C-130 and C-17 carrier of the U.S [17, 18], our research team has established the evaluation criterion for directing the ultra-low airdrop operation. As listed in Table 2, (where $V_{\max }$ is the maximum taxi speed for the landing gear, $V_{\text {minis }}$ minimum level speed for the carrier, $\theta_{\max }$ is the permissible pitch angle to prevent the tail hitting the ground, $\alpha_{\max }$ is the stalling angle, $\theta_{p}, \alpha_{p}$ are trimming pitch angle and angle of attack, 
respectively.) we can make an evaluation of the performance of the aircraft according to the criterion we established.

From the evaluation results about handling quality and mission capacity, we can conclude that the proposed controller is adequate for the airdrop operation in terms of the flight safety and mission achievement.

Table 1 Evaluation result of Chalk Criteria for pitch rate response

\begin{tabular}{|c|c|c|c|}
\hline Index & $\tau_{e}$ & $T P R$ & $\Delta t$ \\
\hline Value & 0.02123 & 0.13208 & 2.2493 \\
\hline Level & $\mathrm{I}$ & $\mathrm{I}$ & $\mathrm{I}$ \\
\hline
\end{tabular}

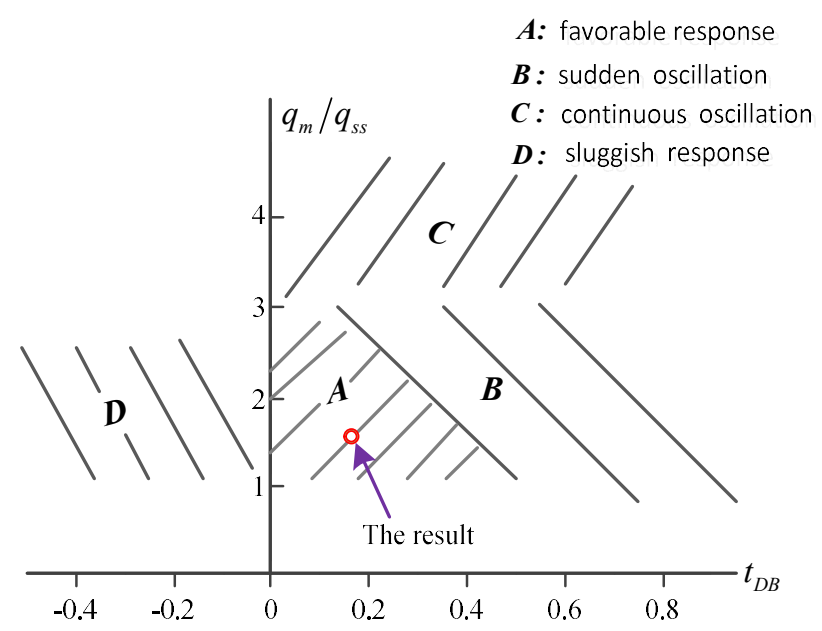

Figure 9. Evaluation result of Gibson Criteria for Pitch angle response

Table 2 Evaluation criterion and result for mission capability and safety

\begin{tabular}{|c|c|c|c|}
\hline \multicolumn{3}{|c|}{ Evaluation criterion of the aircraft for ultra-low airdrop } & \multirow{2}{*}{$\begin{array}{c}\text { Evaluation } \\
\text { result }\end{array}$} \\
\hline Index & Desired range (level.I) & Moderate range (level.II) & \\
\hline$H$ & $H \in[7.2,12]$ & $H \in(3,7.2) \cup(12,30)$ & I \\
\hline$V$ & $\begin{array}{l}V \in\left[0.32 V_{\max }+0.68 V_{\min }\right. \\
\left.0.68 V_{\max }+0.32 V_{\min }\right]\end{array}$ & $\begin{array}{l}V \in\left[0.19 V_{\max }+0.81 V_{\min }\right. \\
\left.0.81 V_{\max }+0.19 V_{\min }\right]\end{array}$ & I \\
\hline$\theta$ & $\Delta \theta_{\max } \in\left(0,0.37\left(\theta_{\max }-\theta_{p}\right)\right]$ & $\Delta \theta_{\max } \in\left[0.37\left(\theta_{\max }-\theta_{p}\right), 0.69\left(\theta_{\max }-\theta_{p}\right)\right]$ & I \\
\hline$\alpha$ & $\Delta \alpha_{\max }\left(0,0.37\left(\alpha_{\max }-\alpha_{p}\right)\right]$ & $\Delta \alpha_{\max }\left(0.37\left(\alpha_{\max }-\alpha_{p}\right), 0.69\left(\alpha_{\max }-\alpha_{p}\right)\right]$ & II \\
\hline
\end{tabular}

\section{Conclusions}

Compared with the normal flight, heavyweight airdrop brings stringent demands for the dynamic response of the aircraft. Some research has been done direct during this complex task:

(1) According to the characteristics of the airdrop operations, the effect of cargo's movement is regarded as a disturbance to the carrier, which simplifies the aerodynamic model. The simulation proves its effectiveness.

(2) In view of the airdrop characteristics that contain strong perturbation and nonlinearity, a novel ADRC control theory is introduced, and the flight controller is designed based on the proposed method to meet the requirement of the airdrop task. Simulation and flight quality evaluation show good performance of the control method in the presence of time-varying aerodynamic coefficients.

(3) The paper provides a pregnant control technique for the thorny problem, benefiting from its advantage that can eliminate the disturbance and decouple control timely independence of the system's accurate mode, which has important significance for reference in an airdrop operation; while further research into the ADRC controller parameter regulating method is still needed.

\section{References}

[1] Desabrais, K. J., Riley, J., Lee, C.: Low-cost high-altitude low-opening cargo airdrop systems, Journal of Aircraft, 49 (2012), 1, 34954.

[2] Zhang, H. Y., Shi, Z. K.: Variable structure control of catastrophic course in airdropping heavy cargo, Chinese Journal of Aeronautics, 22 (2009), 520-527.

[3] Vesna, K., Nino, S.: Improvement of robot trajectory tracking by using nonlinear control methods, Engineering Review, 26 (2006), 7-17.

[4] Chai, L., Qian, C. J.: State feedback stabilization for a class of nonlinear timedelay systems via dynamic linear controller, 
Journal of Systems Science and Complexity, 27 (2014), 3, 453-462.

[5] Feng, Y. L., Shi, Z. K., Tang,W.: Dynamics modeling and control of large transport aircraft in heavy cargo extraction, Journal of Control Theory and Applications, 9 (2011), 2, 231-236.

[6] Abid, S., Chtourou, M., Djemel, M.: Incremental and stable training algorithm for wind turbine neural modeling, Engineering Review, 33 (2013), 3, 165-172.

[7] Han, J. Q.: Active disturbance rejection control technique, National Defense Industry Press, Beijing, 2008.

[8] Zhao, C. Z., Huang, Y.: ADRC based input disturbance rejection for minimum-phase plants with unknown orders and/or uncertain relative degrees, Journal of Systems Science and Complexity, 25 (2012), 4, 625-640.

[9] Liu, R., Sun, X. X., Dong, W. H.: Dynamics modeling and control of a transport aircraft for ultra-low altitude airdrop, Chinese Journal of Aeronautics, 28 (2015), 3, 478-487.

[10] Zhang, J. X., Xu, H. J., Zhang, D. C., Liu, D. L.: Safety modeling and simulation of mutifactor coupling heavy-equipment airdrop, Chinese Journal of Aeronautics, 27 (2014), 5, 1062-1069.

[11] Wang, J., Chen, J., Ouyang, S., Yang, Y.: Trajectory tracking control based on adaptive neural dynamics four-wheel drive omnidirectional mobile robots, Engineering Review, 34 (2014), 3, 235-243.

[12] Zhao, C. Z., Huang, Y.: ADRC based input disturbance rejection for minimum-phase plants with unknown orders and / or uncertain relative degrees, Journal of System Science and Complexity, 25 (2012), 4, 625-640.

[13] Liu, R., Sun, X. X., Dong, W. H.: Dynamics modeling and adaptive control of a transport aircraft for heavy-weight airdrop, Mathematical Problems in Engineering, 2015 (2015), 1-15.

[14] Yang, X. X., Huang, Y.: Capability of extended state observer for estimating uncertainties, Proceedings of the 2009 American Control Conference, Missouri, 2009, 3700-3705.

[15] Bagus, M., Luo, Z. H., Han, J. Q, et al.: Highspeed high-precision motion control of robots using extended states observer. Journal of the Robotios Society of Japan, 2000, 2, 86-93.

[16] U.S. Department Of Defense (2004): Flying qualities of piloted aircraft, U.S Department of Defense Handbook, MIL-STD-1797A.

[17] K. Martin., R. Franzen., R. Ramirez. Evaluation of the C-130E stability and control characteristics and the A/32H-4 dual cargo handling system during low level cargo deliveries, FTC-TR-66-43, 1967.

[18] Kendall E. R.: The design and development of flying qualities for the $\mathrm{C}-17$ military transport airplane, advances in aircraft flight control, 2006, 189-201. 